\title{
Interações medicamentosas em prescrições para idosos hospitalizados com Síndrome Coronariana Aguda
}

Tiago Aparecido Maschio de Lima ${ }^{1}$, Moacir Fernandes de Godoy ${ }^{2}$

\author{
${ }^{1}$ Farmacêutico, Mestre em Enfermagem. \\ Coordenador de Pesquisa Clínica na \\ Fundação Faculdade Regional de Medicina \\ de São José do Rio Preto. Docente na \\ União das Faculdades dos Grandes Lagos. \\ São José do Rio Preto, SP, Brasil. E-mail: \\ tiagomaschio.farmacip@gmail.com. \\ ${ }^{2}$ Médico, Doutor em Cirurgia \\ Cardiovascular. Professor Adjunto na \\ Faculdade de Medicina de São José do Rio \\ Preto. São José do Rio Preto, SP, Brasil. E- \\ mail: mf60204@gmail.com.
}

Recebido: 12/08/2016.

Aceito: 18/05/2017.

Publicado: 01/10/2017

Como citar esse artigo: Lima TAM, Godoy MF. Interações medicamentosas em prescrições para idosos hospitalizados com Síndrome Coronariana Aguda. Rev. Eletr. Enf. [Internet]. 2017 [acesso em: ____19:a24. Disponível em: http://dx.doi.org/10.5216/ree.v19.42764.

\section{RESUMO}

Objetivou-se determinar a taxa de interações medicamentosas potenciais teóricas em prescrições para idosos diagnosticados com Síndrome Coronariana Aguda em um hospital de ensino. Estudo descritivo exploratório com análise de 607 prescrições através de bases de dados informatizadas para identificar e classificar as interações de acordo com a intensidade (maior, moderada ou menor), mecanismo (farmacocinético ou farmacodinâmico) e relevância da documentação. Foram detectadas 10.162 interações, distribuídas entre 554 tipos de combinações diferentes dentre os medicamentos prescritos, e $99 \%$ das prescrições apresentaram pelo menos uma e máximo de 53 interações, destacando-se a prevalência de maiores e moderadas. Houve correlação entre o número de interações e o número de medicamentos prescritos e o tempo de hospitalização. Este estudo contribui para a delimitação do padrão de prevalência das interações medicamentosas em prescrições para Síndrome Coronariana Aguda, além de subsidiar a importância da implantação efetiva da Farmácia Clínica em hospitais de ensino.

Descritores: Prescrições de Medicamentos; Idoso; Cardiologia; Interações de Medicamentos; Hospital de Ensino.

\section{INTRODUÇÃO}

Idosos com Síndrome Coronariana Aguda (SCA) são submetidos ao uso de polifarmácia que configura na utilização de medicamentos de diferentes classes terapêuticas, principalmente quando acometidos por outras doenças concomitantes, cardiovasculares ou não, comuns nessa faixa etária. A polifarmácia acarreta riscos de interações medicamentosas que, na prática clínica representam sérios problemas, podendo, além de causar eventos adversos graves, resultar em ineficácia da terapia medicamentosa ${ }^{(1)}$. 
Define-se interação medicamentosa (IM) como uma resposta farmacológica ou clínica à administração concomitante de dois ou mais fármacos, que seja divergente da resposta desencadeada por esses fármacos quando utilizados isoladamente. De acordo com o mecanismo, são classificadas como farmacocinéticas nas situações em que ocorrem alterações na concentração de pelo menos um dos fármacos envolvidos na interação durante os processos de absorção, distribuição, biotransformação ou eliminação. Enquanto que interações farmacodinâmicas estão relacionadas ao mecanismo de ação dos fármacos envolvidos, geralmente através de antagonismo ou sinergismo. A expressão "Interações Medicamentosas Potenciais Teóricas" (IMPT) descreve interações entre medicamentos presentes na prescrição médica, previamente conhecidas e documentadas, mas que podem ou não ocorrer, exigindo monitoramento clínico e laboratorial ${ }^{(2-3)}$.

O conhecimento das principais características farmacológicas das IM contribui para o seu manejo clínico. Portanto, torna-se fundamental a prevenção de eventos adversos provocados pela presença de IM nas prescrições médicas, através do acesso às bases de dados com informações detalhadas sobre seu mecanismo das IM, classificação de intensidade, orientações de manejo e riscos envolvidos ${ }^{(1,4-5)}$.

São atividades desenvolvidas por farmacêuticos clínicos, a coleta de dados clínicos, identificação de IM e outros problemas relacionados aos medicamentos, monitoramento e manejo de pacientes, contribuindo com a equipe médica para as intervenções clínicas necessárias, melhorando a qualidade da farmacoterapia, e minimizando, consequentemente, os riscos de resultados desfavoráveis da terapia medicamentosa, além de diminuir custos ${ }^{(6-8)}$.

Considerando que as IM configuram entre os fatores responsáveis por prejuízos de saúde e farmacoeconômicos, objetivou-se determinar a taxa e as características das IMPT em prescrições para idosos hospitalizados com diagnóstico de SCA. A escolha deste perfil de pacientes justifica-se pelo consumo de medicamentos pertencentes a diversos grupos farmacológicos para o tratamento da SCA e das doenças concomitantes frequentes ${ }^{(1,7)}$.

\section{MÉTODOS}

Trata-se de um estudo descritivo exploratório. Foram analisadas 607 prescrições de 119 pacientes com diagnóstico de SCA atendidos pelo Sistema Único de Saúde (SUS) da Cardiologia Clínica de um hospital de ensino localizado no interior do Estado de São Paulo, Brasil, entre abril e julho de 2014. Pacientes com idade igual ou acima de 60 anos, independentemente do sexo, foram incluídos no estudo. Foram analisadas as prescrições de cada paciente incluído durante todo o período de hospitalização na unidade.

O hospital do estudo é referência em SCA no município e região, o fato de estar vinculado a uma instituição de ensino e possuir programa de residência médica, aumenta a importância da análise das prescrições pelo farmacêutico clínico. Entretanto, ainda não possui uma equipe de farmacêuticos exclusiva para as atividades clínicas e a Farmácia Clínica está em fase inicial de implantação.

Para avaliação e classificação das IMPT foram utilizadas bases de dados informatizadas Micromedex ${ }^{(9)}$, 
Drugs $^{(10)}$ e Medscape ${ }^{(11)}$. As IMPT foram classificadas de acordo com o nível de intensidade em:

Maior, contraindicada, importante ou séria (quando a interação representa risco à vida e/ou requerer intervenção médica para diminuir ou evitar efeitos graves ou os medicamentos são contraindicados para uso concomitante);

Moderada ou significante (quando a interação resulta em exacerbação do problema de saúde do paciente e/ou requer uma alteração na farmacoterapia);

Menor ou secundária (quando a interação resulta em efeitos clínicos limitados. As manifestações podem incluir um aumento na frequência ou intensidade dos efeitos adversos, mas geralmente não requerem uma alteração importante na farmacoterapia $)^{(9-11)}$.

A base de dados do Micromedex é baseada em evidências científicas e amplamente utilizada em muitos países. As bases de dados do Drugs e Medscape foram incluídas neste estudo pela disponibilidade online gratuita, tornando-se importantes fontes de informação na saúde pública ${ }^{(4-5,9-11)}$. Nos casos de discordância entre a classificação pelas bases, a maior intensidade foi considerada. As IMPT também foram classificadas pelo perfil farmacocinético ou farmacodinâmico. Na análise de IMPT, também foram incluídas as interações consideradas positivas ou intencionais, que são aquelas que proporcionam benefício através de seu efeito sinérgico. Foram excluídos os fitoterápicos, eletrólitos da soroterapia e componentes da dieta ${ }^{4-}$ 5).

Além disso, as interações foram classificadas de acordo com a relevância da documentação em:

- Excelente (nos casos em que os estudos controlados estabeleceram de modo claro a existência da interação);

- Boa (nos casos em que a documentação sugere com veemência a existência da interação, mas faltam estudos controlados realizados de modo adequado);

- Razoável (quando a documentação disponível é insatisfatória, mas as considerações farmacológicas levam os clínicos a suspeitar da existência da interação; ou a documentação é boa para um medicamento farmacologicamente similar);

- Desconhecida ${ }^{(9)}$.

Avaliou-se a correlação entre IMPT com idade, número de medicamentos prescritos, e tempo de hospitalização dos participantes, através do coeficiente de correlação de Pearson. Também foi promovida uma análise descritiva visando caracterizar as interações medicamentosas. Variáveis discretas e não contínuas foram apresentadas como mediana, intervalo mínimo e máximo. As variáveis categóricas são apresentadas como números absolutos e proporções (\%). Em todas as circunstâncias, um valor de $p<0,05$ foi considerado estatisticamente relevante. SPSS Statistics versão 22.0 foi utilizado para as análises.

A pesquisa foi aprovada pelo Comitê de Ética em Pesquisa (CEP) da Faculdade de Medicina de São José do Rio Preto (Famerp), sob o parecer no 613.171. O diagnóstico de SCA foi confirmado por meio do prontuário eletrônico dos participantes, e a consulta das prescrições médicas ocorreu da mesma forma. 


\section{RESULTADOS}

Todas as informações referentes às prescrições analisadas foram transpostas para uma planilha do software Microsoft ${ }^{\bullet}$ Excel (2010), atualizada constantemente durante o estudo por meio de consultas frequentes às bases de dados informatizadas ${ }^{(9-11)}$. Ao final da coleta de dados a planilha foi atualizada e corrigida, contando com informações de 145 diferentes tipos de medicamentos prescritos, quantitativo de 7.266 e um total de 10.162 IMPT relacionadas a estes medicamentos, distribuídas entre 554 tipos de combinações diferentes entre medicamentos prescritos. A mediana foi 15, número mínimo de uma e máximo de 53 IMPT por prescrição. A Tabela 1 caracteriza o perfil categórico das IMPT observadas nas prescrições analisadas.

Tabela 1: Distribuição por nível de intensidade das Interações Medicamentosas Potenciais Teóricas. São José do Rio Preto, SP, Brasil, 2014.

\begin{tabular}{|c|c|c|c|c|}
\hline \multirow{2}{*}{ Nível de intensidade } & \multicolumn{2}{|c|}{ Total } & \multicolumn{2}{|c|}{ Tipos de combinação } \\
\hline & $\mathbf{n}$ & $\%$ & $\mathrm{n}$ & $\%$ \\
\hline Maior & 2566 & 25 & 124 & 22 \\
\hline Moderado & 6504 & 64 & 372 & 68 \\
\hline Menor & 1092 & 11 & 58 & 10 \\
\hline Total & 10162 & 100 & 544 & 100 \\
\hline
\end{tabular}

Entre as prescrições analisadas, $99 \%$ apresentavam pelo menos uma IMPT destacando-se a prevalência daquelas moderadas e maiores, $64 \%$ e 25\%, respectivamente. A Figura 1 apresenta a relação de porcentagem de prescrições em que foram observadas as 20 IMPT mais frequentes no período de análise de dados. Quanto ao mecanismo de interação, $73 \%$ das IMPT foram consideradas farmacodinâmicas e $27 \%$ farmacocinéticas.

A Tabela 2 lista as principais características das 14 IMPT maiores frequentemente observadas no estudo. A Tabela 3 apresenta as características referentes as 14 IMPT moderadas mais frequentes no estudo. 
IMPT* Maior IMPT*Moderada IMPT*Menor

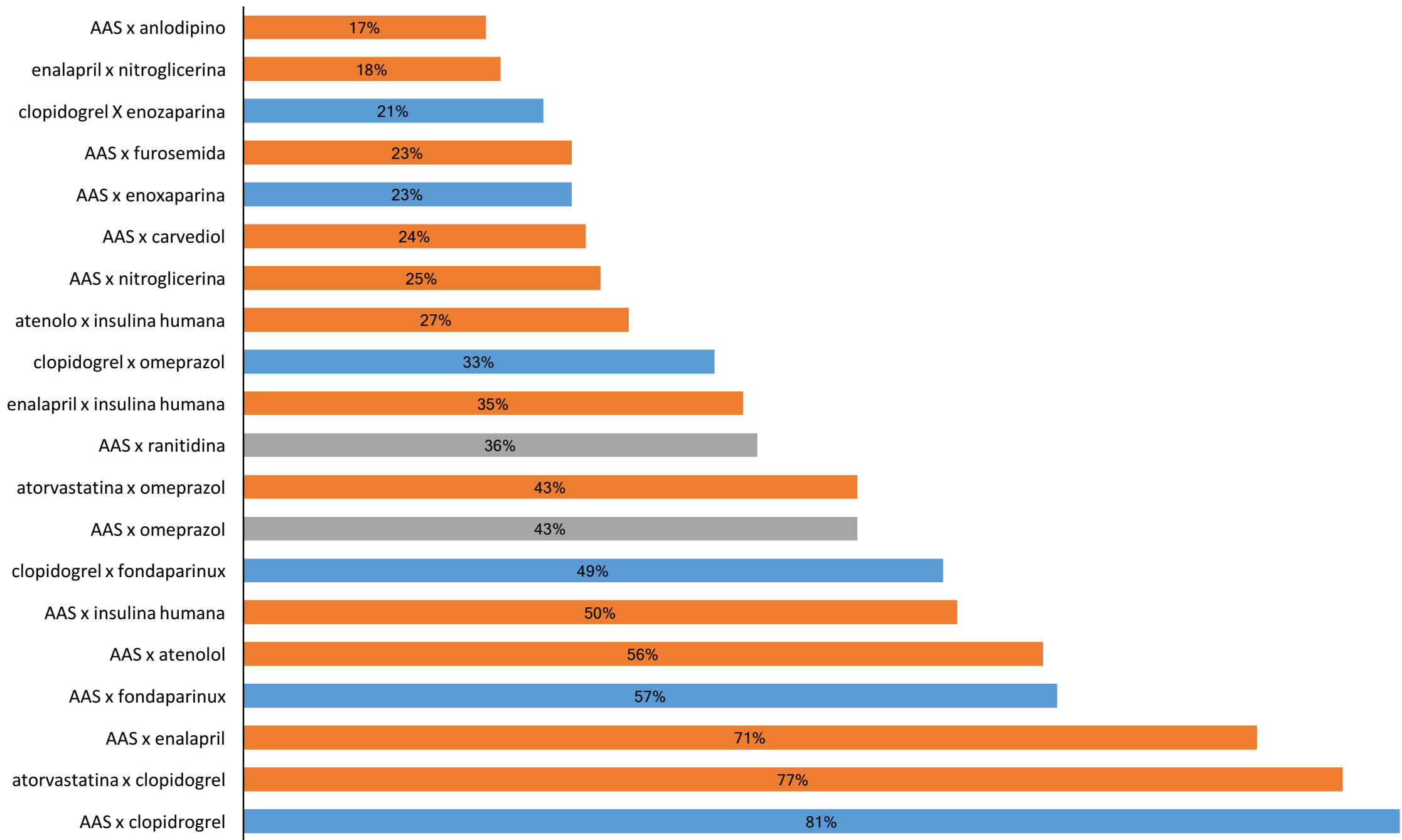

*IMPT: Interações medicamentosas potenciais teóricas

Figura 1: Tipos de IMPT prevalentes no estudo e sua frequência no total de prescrições analisadas. São José do Rio Preto, SP, Brasil, 2014.

Rev. Eletr. Enf. [Internet]. 2017 [acesso em:_____];19:a24. Disponível em: http://dx.doi.org/10.5216/ree.v19.42764. 
Tabela 2: Características e frequência das IMPT Maiores prevalentes nas prescrições estudadas. São José do Rio Preto, SP, Brasil, 2014.

\begin{tabular}{|c|c|c|c|c|c|c|c|c|}
\hline \multirow{3}{*}{$\begin{array}{c}\text { IMPT } \\
\text { AAS/clopidogrel }\end{array}$} & \multirow{3}{*}{$\begin{array}{c}\text { Evento } \\
\text { Aumento do risco de sangramento }\end{array}$} & \multirow{3}{*}{$\begin{array}{c}\text { Provável Mecanismo Geral } \\
\text { Farmacodinâmica }\end{array}$} & \multicolumn{3}{|c|}{ Base de dados* } & \multirow{3}{*}{$\begin{array}{c}\text { Documentação } \\
\text { Razoável }\end{array}$} & \multicolumn{2}{|c|}{$\begin{array}{l}\text { Frequência nas } \\
\text { prescrições }\end{array}$} \\
\hline & & & & & & & \multirow{2}{*}{\begin{tabular}{c|c}
$n$ \\
493
\end{tabular}} & \multirow{2}{*}{$\begin{array}{l}\% \\
81\end{array}$} \\
\hline & & & 1 & 2 & 3 & & & \\
\hline AAS/fondaparinux & Aumento do risco de sangramento & Farmacodinâmica & 1 & 2 & 3 & Razoável & 344 & 57 \\
\hline clopidogrel/fondaparinux & Aumento do risco de sangramento & Farmacodinâmica & 1 & 2 & 3 & Razoável & 298 & 49 \\
\hline clopidogrel/omeprazol & $\begin{array}{l}\text { Risco de trombose pela redução da formação do metabólito ativo do } \\
\text { clopidogrel por inibição da CYP2C19 }\end{array}$ & Farmacocinética & 1 & 2 & 3 & Excelente & 200 & 33 \\
\hline AAS/enoxaparina & Aumento do risco de sangramento & Farmacodinâmica & 1 & 2 & 3 & Boa & 142 & 23 \\
\hline clopidogrel/enoxaparina & Aumento do risco de sangramento & Farmacodinâmica & 1 & 2 & 3 & Razoável & 125 & 21 \\
\hline anlodipino/clopidogrel & Redução do efeito antiplaquetário e risco de eventos trombóticos & Farmacocinética & 1 & NC & NC & Excelente & 84 & 14 \\
\hline AAS/heparina & Aumento do risco de sangramento & Farmacodinâmica & 1 & 2 & 3 & Razoável & 58 & 10 \\
\hline clopidogrel/heparina & Aumento do risco de sangramento & Farmacodinâmica & 1 & 2 & 3 & Razoável & 38 & 6 \\
\hline atorvastatina/claritromicina & $\begin{array}{l}\text { Risco de miopatia e rabdomiólise pelo aumento do nível sérico da } \\
\text { atorvastatina por inibição enzimática da CYP3A4 }\end{array}$ & Farmacocinética & 1 & 2 & 3 & Boa & 36 & 6 \\
\hline claritromicina/clopidogrel & $\begin{array}{l}\text { Redução na formação do metabólito ativo do clopidogrel por inibição da } \\
\text { CYP3A4, resultando em alta atividade plaquetária }\end{array}$ & Farmacocinética & NC & 2 & 3 & Não classificada & 35 & 6 \\
\hline fondaparinux/levotiroxina & Aumento do efeito do fondaparinux & Farmacodinâmica & NC & NC & 3 & Não classificada & 33 & 5 \\
\hline enalapril/espironolactona & Risco de hipercalemia & Farmacodinâmica & 1 & 2 & 3 & Boa & 25 & 4 \\
\hline AAScitalopram & Aumento do risco de sangramento & Farmacodinâmica & 1 & 2 & 3 & Boa & 24 & 4 \\
\hline
\end{tabular}

* Bases de dados: (1) Micromedex, (2) Drugs, (3) Medscape

** NC: Não classificada pela base de dados 
Tabela 3: Características e frequência das IMPT Moderadas prevalentes nas prescrições estudadas. São José do Rio Preto, SP, Brasil, 2014.

\begin{tabular}{|c|c|c|c|c|c|c|c|c|}
\hline \multirow[t]{2}{*}{ IMPT } & \multirow[t]{2}{*}{ Evento } & \multirow{3}{*}{$\begin{array}{c}\text { Provável } \\
\text { Mecanismo }\end{array}$} & \multirow{2}{*}{\multicolumn{3}{|c|}{$\begin{array}{l}\text { Base de } \\
\text { dados* }\end{array}$}} & \multirow[t]{2}{*}{ Documentação } & \multicolumn{2}{|c|}{$\begin{array}{c}\text { Frequência nas } \\
\text { prescrições }\end{array}$} \\
\hline & & & & & & & $n$ & $\%$ \\
\hline atorvastatina/clopidogrel & $\begin{array}{l}\text { Redução da formação do metabólito ativo do clopidogrel por inibição da CYP3A4, resultando } \\
\text { em alta atividade plaquetária }\end{array}$ & & 1 & 2 & NC & Excelente & 465 & 77 \\
\hline AAS/enalapril & Redução do efeito anti-hipertensivo & Farmacodinâmica & 1 & 2 & 3 & Excelente & 430 & 71 \\
\hline AAS/atenolol & Redução do efeito anti-hipertensivo & Farmacodinâmica & 1 & 2 & 3 & Boa & 339 & 56 \\
\hline AAS/insulina humana & Risco de hipoglicemia & Farmacodinâmica & 1 & 2 & 3 & Razoável & 305 & 50 \\
\hline atorvastatina/omeprazol & $\begin{array}{c}\text { Risco de miopatia e rabdomiólise pelo aumento do nível sérico da atorvastatina por inibição } \\
\text { enzimática da CYP3A4 e glicoproteína P }\end{array}$ & Farmacocinética & NC & 2 & NC & $\begin{array}{c}\text { Não } \\
\text { classificada }\end{array}$ & 262 & 43 \\
\hline $\begin{array}{l}\text { enalapril/insulina } \\
\text { humana }\end{array}$ & Risco de hipoglicemia & Farmacodinâmica & 1 & 2 & 3 & Razoável & 215 & 35 \\
\hline atenolol/insulina humana & Risco de hipoglicemia, hiperglicemia e hipertensão & Farmacodinâmica & 1 & 2 & NC & Boa & 165 & 27 \\
\hline AAS/nitroglicerina & $\begin{array}{l}\text { Aumento no nível sérico da nitroglicerina e efeito aditivo na } \\
\text { depressão plaquetária }\end{array}$ & Farmacodinâmica & 1 & 2 & NC & Boa & 151 & 25 \\
\hline AAS/carvedilol & Redução do efeito anti-hipertensivo & Farmacodinâmica & 1 & 2 & 3 & Boa & 148 & 24 \\
\hline AAS/furosemida & Redução do efeito diurético e anti-hipertensivo & Farmacodinâmica & 1 & 2 & 3 & Boa & 138 & 23 \\
\hline enalapril/nitroglicerina & Aumento dos efeitos hipotensores da nitroglicerina & Farmacodinâmica & NC & 2 & NC & $\begin{array}{c}\text { Não } \\
\text { classificada }\end{array}$ & 109 & 18 \\
\hline AAS/anlodipino & Aumento do risco de hemorragia gastrointestinal e antagonismo do efeito hipotensor & Farmacodinâmica & 1 & 2 & NC & Boa & 102 & 17 \\
\hline AAS/losartana & Redução no efeito anti-hipertensivo e risco de insuficiência renal & Farmacodinâmica & 1 & 2 & 3 & Boa & 89 & 15 \\
\hline enalapril/enoxaparina & Risco de hipercalemia & Farmacodinâmica & NC & 2 & NC & $\begin{array}{l}\text { Não } \\
\text { classificada }\end{array}$ & 89 & 15 \\
\hline
\end{tabular}

* Bases de dados: (1) Micromedex, (2) Drugs, (3) Medscape

** NC: Não classificada pela base de dados 
Foi observada correlação positiva estatisticamente significante entre o número de IMPT e o tempo de hospitalização (Tabela 4). Destaca-se também, a correlação positiva entre o número de IMPT com o número de medicamentos prescritos (Tabela 4). Não houve correlação estatisticamente significante entre as IMPT e a idade dos pacientes (Tabela 4).

Tabela 4: Correlação do número de IMPT, total e por intensidade, com a idade dos pacientes, seu tempo de hospitalização e o número de medicamentos prescritos. São José do Rio Preto, SP, Brasil, 2014.

\begin{tabular}{|c|c|c|c|c|}
\hline & Total & IMPT & IMPT & IMPT \\
\hline & IMPT (119)** & Maiores (119)** & Moderadas (118)** & Menores (95)** \\
\hline \multirow{2}{*}{ Idade } & $r=0,1523$ & $r=0,1728$ & $r=0,1377$ & $r=0,1444$ \\
\hline & $P=0,0982$ & $P=0,0603$ & $P=0,1350$ & $P=0,1168$ \\
\hline \multirow{2}{*}{ Dias de hospitalização } & $r=\underline{0,9045}$ & $r=\underline{0,9235}$ & $r=\underline{0,9235}$ & $r=\underline{0,7809}$ \\
\hline & $P=<0,0001$ & $P=<0,0001$ & $P=<0,0001$ & $P=<0,0001$ \\
\hline \multirow{2}{*}{ Número de medicamentos } & $r=\underline{0,9719}$ & $r=\underline{0,9626}$ & $r=\underline{0,9510}$ & $r=\underline{0,8482}$ \\
\hline & $P=<0,0001$ & $P=<0,0001$ & $P=<0,0001$ & $P=<0,0001$ \\
\hline
\end{tabular}

* Rho=coeficiente de correlação de Pearson; $P=$ valor- $P$; $n=$ número de sujeitos ( $n=119)$.

** número de pacientes expostos à IMPT da classe indicada.

\section{DISCUSSÃO}

Os resultados obtidos neste estudo permitem avaliar os potenciais riscos envolvendo prescrições hospitalares destinadas à SCA. Sabe-se que, pela complexidade da síndrome e pelo número elevado de medicamentos utilizados, e ainda considerando a terapia utilizada nas suas comorbidades, suas prescrições são mais predispostas a apresentar IMPT. Esse perfil de pacientes também se encontra mais exposto à ocorrência de eventos adversos aos medicamentos, fato associado a fatores como o número de medicamentos administrados, a complexidade dos esquemas terapêuticos, e ao estado clínico do paciente. Ainda, vale ressaltar que os problemas de prescrição da farmacoterapia são bastante comuns em hospitais de ensino, reforçando a importância da atuação do farmacêutico clínico na análise das prescrições ${ }^{(11-14)}$.

Com uma parcela de $99 \%$ das prescrições apresentando ao menos uma IMPT, fica evidente a necessidade de avaliação e acompanhamento das prescrições sem negligenciar os riscos das interações potenciais. O elevado número de IMPT observadas nos resultados corrobora com a taxa obtida em outros estudos, que apontam este fato comum às prescrições de cardiologia. Apesar da relevância dessa informação por reforçar o potencial risco inerente às IMPT, cabe ressaltar que esse número inclui todas as classes de IMPT, incluindo também aquelas consideradas intencionais e positivas na farmacoterapia para SCA ${ }^{(1,12-13)}$.

A IMPT mais frequente neste estudo foi entre ácido acetilsalicílico (AAS) e clopidogrel com nível de intensidade maior. A orientação de manejo clínico dessa interação recomenda que o uso concomitante dos dois fármacos deve ser feito com cautela mediante monitoramento contínuo quanto ao risco de sangramento. A mesma recomendação de manejo é válida para as outras interações maiores, detectadas neste estudo, que envolvem a associação entre antiplaquetários, como AAS e clopidogrel, e anticoagulantes, como enoxaparina e fondaparinux. A associação desses fármacos é recomendada pelos Protocolos Clínicos e nas Diretrizes Terapêuticas, internacionais e nacionais, para SCA e, embora essas interações aumentem o risco de sangramento, são também consideradas positivas e intencionais, pelo benefício comprovado na 
redução de eventos cardiovasculares em pacientes com SCA ${ }^{(15-18)}$.

Outra IMPT de intensidade maior frequentemente encontrada nas prescrições analisadas foi a interação entre clopidogrel e omeprazol. O clopidogrel é um pró-fármaco metabolizado no fígado pela CYP2C19 para gerar seu metabólito ativo e adquirir suas propriedades antiagregantes plaquetárias. O omeprazol é inibidor enzimático da enzima ativadora do clopidogrel, provocando diminuição da efetividade da terapia antiplaquetária pela inibição da conversão de seu metabólito ativo. Alguns estudos mostraram-se controversos na utilização concomitante do clopidogrel e omeprazol. A recomendação é a contraindicação da utilização concomitante dos dois fármacos. O manejo recomendado é a substituição do omeprazol por outro inibidor de bomba de prótons que não atue no mesmo citocromo ${ }^{(19-21)}$. Os medicamentos que reduzem a acidez, por exemplo, os antagonistas do receptor $\mathrm{H} 2$, podem reduzir a incidência de sangramento gastrointestinal em pacientes com SCA que são tratados com antiplaquetários e, a menos que contraindicado, o seu uso deve ser considerado. O uso de um inibidor da bomba de prótons é permitido, exceto pelo uso concomitante de omeprazol ou esomeprazol com clopidogrel ${ }^{(2,15)}$.

Dentro desse contexto, todas as interações de severidade maior ou moderada foram notificadas à equipe médica responsável pelas prescrições, através de intervenções pontuais por alertas verbais. A postura adotada pela equipe diante dos alertas referentes à IMPT correspondeu as orientações de manejo recomendadas, principalmente quanto ao monitoramento contínuo devido ao risco de sangramento nas IMPT que envolvem a associação entre antiplaquetários e anticoagulantes, e substituição do omeprazol por ranitidina quando associado ao clopidogrel ${ }^{(2,15,19-21)}$.

As IMPT de intensidade moderada são as mais comuns entre as interações medicamentosas e consequentemente configuram entre as mais frequentes na maioria dos estudos que avaliam as interações presentes nas prescrições. Neste estudo, destacam-se as interações moderadas envolvendo fármacos antihipertensivos podendo ocorrer hipotensão ou redução no efeito anti-hipertensivo, sendo que essa última situação favorece o quadro de SCA ${ }^{(9-11,16-18)}$. Nesses casos, o farmacêutico clínico deverá lançar o alerta de interação potencial, podendo utilizar prontuário eletrônico como ferramenta, e em parceria com a equipe médica, estabelecer a decisão conjunta de manutenção ou alteração da terapia medicamentosa baseandose na clínica dos pacientes e nos resultados laboratoriais. Nos casos de alteração de concentração plasmática causados pelas IMPT é possível, para alguns fármacos, verificar sua concentração sérica através de exames laboratoriais e assim observar se a interação está efetivamente ocorrendo ou não, porém essa rotina envolve custos e recursos humanos, e ainda não faz parte da rotina da maioria dos hospitais de ensino brasileiros, assim como no hospital deste estudo ${ }^{(7,12-14,22)}$.

O uso de sinalização das interações através de alertas no prontuário informatizado, bloqueio da prescrição eletrônica na presença de interações maiores e a intervenção farmacêutica diária em parceria com a equipe médica, são estratégias eficientes na redução de IMPT e seus problemas relacionados ${ }^{(23-24)}$.

Estudos com foco na elucidação dos riscos e benefícios da terapia medicamentosa pelo farmacêutico clínico, assim como este estudo, representam uma importante contribuição para medidas de rastreamento 
de eventos adversos a medicamentos e otimização da farmacoterapia preconizada pelos protocolos clínicos. Recomenda-se a utilização de no mínimo três fontes de informação para a pesquisa de IMPT, e a análise crítica dessas fontes disponíveis na literatura, incluindo as bases de dados informatizadas, para que não ocorra uma subidentificação. Sugere-se a implantação das três bases de dados utilizadas neste estudo. A partir do conhecimento das IMPT de cada prescrição, é possível identificar a ocorrência de interações reais através de acompanhamento das visitas ao leito, avaliação de prescrições e discussões de protocolos ${ }^{(4-5,7-8,25)}$.

Os padrões observados neste estudo cooperam para o delineamento do perfil de IMPT prevalentes em unidades cardiológicas brasileiras e demonstra necessidade do desenvolvimento de ações preventivas sistematizadas. Os resultados apresentados corroboram com outros estudos realizados em hospitais de ensino, como a prevalência de interações moderadas e maiores e a correlação entre o número de medicamentos prescritos e o tempo de hospitalização com a presença de IMPT em prescrições ${ }^{(12-14)}$.

Outro fato conhecido na literatura, através de estudos com diferentes desenhos e tamanhos amostrais variados, é a relação entre o número de medicamentos prescritos e o número de IMPT presentes em prescrição. Essa correlação realça o risco intrínseco à polifarmácia presente nas prescrições envolvendo elevado arsenal de medicamentos ${ }^{(1,7-8,12-14)}$.

Pelo fato do hospital de ensino deste estudo não possuir um serviço de Farmácia Clínica formado e atuante, não observou-se a ocorrência real das interações nos pacientes, sendo este um fator limitante, portanto, para futuros estudos sugere-se o acompanhamento contínuo do grupo de pesquisa para avaliação da incidência de ocorrências clínicas relacionadas à IMPT, através de ações contínuas de Farmácia Clínica.

\section{CONCLUSÃO}

Observa-se elevada taxa de interações potenciais teóricas nas prescrições para idosos hospitalizados por Síndrome Coronariana Aguda. Para o tratamento da Síndrome Coronariana Aguda e das doenças concomitantes, comuns nessa faixa etária, esse perfil de pacientes consome medicamentos pertencentes a diversos grupos farmacológicos acarretando interações medicamentosas. Quase todas as prescrições apresentaram pelo menos uma interação medicamentosa. As interações de intensidade moderada e maior foram as mais frequentes, e a maioria envolvia mecanismo farmacodinâmico.

Este trabalho fornece uma colaboração significativa para a delimitação do padrão de prevalência das interações medicamentosas potenciais teóricas nas prescrições para Síndrome Coronariana Aguda, contribuindo para a produção e revisão de estratégias de identificação e manejo através de intervenções farmacêuticas.

Espera-se que este estudo motive a produção de mais pesquisas envolvendo o tema nos hospitais de ensino, e que sirva de subsídio para a implantação efetiva da Farmácia Clínica, visto que a atuação de farmacêuticos clínicos contribui para alcançar melhores resultados farmacoterapêuticos. 


\section{REFERÊNCIAS}

1. Murtaza G, Khan MYG, Azhar S, Khan SA, Khan TM. Assessment of potential drug-drug interactions and its associated factors in the hospitalized cardiac patients. Saudi Pharm J [Internet]. 2016 [acesso em: 25 set. 2017];24:220-5. Disponível em: http://dx.doi.org/10.1016/j.jsps.2015.03.009.

2. Brunton L, Chabner BA, Knollmann B. Goodman and Gilman's Pharmacological Basis of Therapeutics. 12a Edition. New York: McGraw-Hill; 2012.

3. Armaganijan D, Timerman A. Interações medicamentosas: definições e mecanismos. Rev. Soc. Cardiol. Estado de São Paulo. 2013;23(3):11-6.

4. Ayvaz S, Horn J, Hassanzadeh O, Zhu Q, Stan J, Tatonetti NP, et al. Toward a complete dataset of drug-drug interaction information from publicly available sources. J Biomed Inform [Internet]. 2015 [acesso em: 25 set. 2017];55:206-17. Disponível em: http://dx.doi.org/10.1016/j.jbi.2015.04.006.

5. Kim S, Liu H, Yeganova L, Wilbur WJ. Extracting drug-drug interactions from literature using a rich feature-based linear kernel approach. J Biomed Inform [Internet]. 2015 [acesso em: 25 set. 2017];55:23-30. Disponível em: http://dx.doi.org/10.1016/j.jbi.2015.03.002.

6. American College of Clinical Pharmacy. The definition of clinical pharmacy. Pharmacotherapy [Internet]. 2008 [acesso em: 25 set. 2017];28(6):816-7. Disponível em: http://dx.doi.org/10.1592/phco.28.6.816.

7. Cai H, Dai H, Hu Y, Yan X, Xu H. Pharmacist care and the management of coronary heart disease: a systematic review of randomized controlled trials. BMC Health Services Research [Internet]. 2013 [acesso em: 25 set. 2017];13:461.

Disponível em: https://doi.org/10.1186/1472-6963-13-461

8. Zhai XB, Tian DD, Liu XY. The role of the clinical pharmacist in reducing mortality in hospitalized cardiac patients: a prospective, nonrandomized controlled trial using propensity score methods. Int J Clin Pharmacol Ther [Internet]. 2015 [acesso em: 25 set. 2017];53(3):220-9. Disponível em: http://dx.doi.org/10.5414/CP202111.

9. Micromedex [Internet]. Michigan (EUA): Truven Health Analytics. c2017 [acesso em: 25 set. 2017]. Disponível em: http://www-micromedexsolutions-

com.ez58.periodicos.capes.gov.br/micromedex2/librarian/ND_T/evidencexpert/ND_PR/evidencexpert/CS/E56921/ND _AppProduct/evidencexpert/DUPLICATIONSHIELDSYNC/A8B3F8/ND_PG/evidencexpert/ND_B/evidencexpert/ND_P/e videncexpert/PFActionId/evidencexpert.FindDrugInteractions?navitem=top/nteractions.

10. Drug Interactions Checker [Internet]. Auckland (NZ): Drugs.com. c2017 [acesso em: 25 set. 2017]. Disponível em: https://www.drugs.com/drug_interactions.php.

11. Drug Interaction Checker [Internet]. Nova lorque (EUA): Medscape. c1994-2017 [acesso em: 25 set. 2017$].$ Disponível em: http://reference.medscape.com/drug-interactionchecker.

12. Patel VK, Acharya LD, Rajakannan T, Mallayasamy S, Guddttu V, Padmakumar R. Potential drug interactions in patients admitted to cardiology wards of a south Indian teaching hospital. Australas Med J [Internet]. 2011 [acesso em: 25 set. 2017];4(1):9-14. Disponível em: https://dx.doi.org/10.4066/AMJ.2011.450.

13. Sharma S, Chhetri HP, Alam K. A study of potential drug-drug interactions among hospitalized cardiac patients in a teaching hospital in Western Nepal. Indian J Pharmacol [Internet]. 2014 [acesso em: 25 set. 2017];46(2):152-6. Disponível em: http:dx.doi.org/10.4103/0253-7613.129303.

14. Ellitt GR, Engblom E, Aslani P, Westerlund T, Chen TF. Drug related problems after discharge from an Australian teaching hospital. Pharm World Sci [Internet]. 2010 [acesso em: 25 set. 2017];32(5):622-30. Disponível em:

http:dx.doi.org/10.1007/s11096-010-9406-9.

15. Lorga Filho AM, Azmus AD, Soeiro AM, Quadros AS, Avezum Junior A, Marques AC et al. Diretrizes brasileiras de antiagregantes plaquetários e anticoagulantes em cardiologia. Arq Bras Cardiol [Internet]. 2013 [acesso em: 25 set. 2017];101(3 Suppl. 3):1-95. Disponível em: http://dx.doi.org/10.5935/abc.2013S009.

16. Nicolau JC, Timerman A, Marin-Neto JA, Piegas LS, Barbosa CJDG, Franci A et al. Diretrizes da Sociedade Brasileira de Cardiologia sobre Angina Instável e Infarto Agudo do Miocárdio sem Supradesnível do Segmento ST (II Edição, 2007) - Atualização 2013/2014. Arq Bras Cardiol [Internet]. 2014 [acesso em: 25 set. 2017];102(3 Suppl 1):1-75. Disponível em: http://dx.doi.org/10.5935/abc.2014S001.

17. O'Gara PT, Kushner FG, Ascheim DD, Casey Jr DE, Chung MK, Lemos JA et al. 2013 ACCF/AHA guideline for the management of ST-elevation myocardial infarction: a report of the American College of Cardiology Foundation/American Heart Association Task Force on Practice Guidelines. Circulation [Internet]. 2013 [acesso em: 25 set. 2017];127(4):e362-425. Disponível em: http://dx.doi.org/10.1161/CIR.0b013e3182742cf6. 
18. Amsterdam EA, Wenger NK, Brindis RG, Casey Jr DE, Ganiats TG, Holmes Jr DR et al. 2014 AHA/ACC Guideline for the Management of Patients with Non-ST-Elevation Acute Coronary Syndromes: a report of the American College of Cardiology/American Heart Association Task Force on Practice Guidelines. J Am Coll Cardiol [Internet]. 2014 [acesso em: 25 set. 2017];64(24):e139-228. Disponível em: http://dx.doi.org/10.1016/j.jacc.2014.09.017.

19. Siller-Matula JM, Trenk D, KrähenbühI S, Michelson AD, Delle-Karth G. Clinical implications of drug-drug interactions with P2Y12 receptor inhibitors. J Thromb Haemost [Internet]. 2014 [acesso em: 25 set. 2017];12(1):2-13. Disponível em: http://dx.doi.org/10.1111/jth.12445.

20. Aquino JPA, Brophy JM. The potential interaction between clopidogrel and proton pump inhibitors: a systematic review. BMC Med [Internet]. 2010 [acesso em: 25 set. 2017];8:81. Disponível em: http://dx.doi.org/10.1186/17417015-8-81.

21. Casado-Arroyo R, Muñoz-Villalenguas M, Lanas Arbeloa A. Antiagregantes plaquetarios e inhibidores de la bomba de protones. ¿Cómo optimizar el riesgo-beneficio en los pacientes con riesgo cardiovascular y riesgo de hemorragia gastrointestinal? Gastroenterol Hepatol [Internet]. 2011 [acesso em: 25 set. 2017];34(7):478-91. Disponível em: http://dx.doi.org/10.1016/j.gastrohep.2011.04.002.

22.Duan JZ, Jackson AJ, Zhao P. Bioavailability considerations in evaluating drug-drug interactions using the population pharmacokinetic approach. J Clin Pharmacol [Internet]. 2011 [acesso em: 25 set. 2017];51(7):1087-100. Disponível em: http://dx.doi.org/10.1177/0091270010377200.

23. Moura CS, Prado NM, Belo NO, Acurcio FA. Evaluation of drug-drug interaction screening software combined with pharmacist intervention. Int J Clin Pharm [Internet]. 2012 [acesso em: 25 set. 2017];34(4):547-52. Disponível em: http://dx.doi.org/10.1007/s11096-012-9642-2.

24. Barrueco N, Fernández-llamazares CM, Carlavilla EP. Ayudas a la prescripción: interacciones de medicamentos. Acta Pediatr Esp [Internet]. 2008 [acesso em: 25 set. 2017];66(6):277-85. Disponível em:

http://www.actapediatrica.com/index.php/secciones/originales/537-ayudas-a-la-prescripci\%C3\%B3n-interaccionesde-medicamentos

25. Reis WCT, Scopel CT, Correr CJ, Andrzejevski VMS. Analysis of clinical pharmacist interventions in a tertiary teaching hospital in Brazil. Einstein (São Paulo) [Internet]. 2013 [acesso em: 25 set. 2017];11(2):190-6. Disponível em: http://dx.doi.org/10.1590/S1679-45082013000200010. 